\title{
ON THE DIMENSION OF MODULES AND ALGEBRAS IX \\ DIRECT LIMITS
}

\author{
ISRAEL BERSTEIN
}

Let $J$ be a directed set and let $\left\{\Lambda_{j}, \varphi_{i j}\right\}$ be a direct system of rings indexed by $J$ and with limit $\Lambda$. Let $\left\{A_{j}, \psi_{i j}\right\}$ be a direct system of groups indexed by $J$. Assume that each $A_{j}$ is a left $\lambda_{j}$-module and that $\psi_{i j}(\lambda a)=\varphi_{i j}(\lambda) \psi_{i j}(a)$ for each $\lambda \in A_{j}, a \in A_{j}$. Then the limit $A$ of $\left\{A_{j}, \psi_{i j}\right\}$ is a left $A$-module.

THEOREM. If $J$ is countable, then

$$
\text { 1. } \operatorname{dim}_{\Lambda} A \leqq 1+\sup _{j} .1 . \operatorname{dim}_{\Lambda_{j}} A_{j} .
$$

Corollary 1. If $J$ is countable, then

$$
\text { 1. gl. } \operatorname{dim} A \leqq 1+\sup _{j} \text {. l.gl. } \operatorname{dim} . l_{j}
$$

Corollary 2. Let $\left\{K_{j}, \nu_{i j}\right\}$ be a direct systen of commutative rings indexed by $J$ and with limit $K$. Assume that each $\alpha_{j}$ is a $K_{j}$-algebra and that $\varphi_{i j}(k \lambda)$ $=\nu_{i j}(k) \varphi_{i j}(\lambda)$ for $k \in K_{j}, \lambda \in \Lambda_{j}$. Then 1 is a K-algebra. If $J$ is countable, then

$$
K-\operatorname{dim} A \leqq 1+\sup . K_{j}-\operatorname{dim} A_{j}
$$

To derive Cor. 2 we note that

$$
K \cdot \operatorname{dim} A=1 \cdot \operatorname{dim}_{\Lambda^{e}} A, \text { where } A^{e}=\Lambda \otimes_{K} A^{*},
$$

and that $A^{e}$ is the direct limit of $\left\{A_{j}^{e}\right\}$. Cor. 2 is a generalization of a theorem by Kuročkin [1] (see also [2], p. 92).

Proof of the Theorem. We consider the exact sequences

$$
\begin{aligned}
& 0 \longrightarrow R_{j} \longrightarrow F_{j} \rightarrow A_{j} \longrightarrow 0, \quad j \in J \\
& 0 \longrightarrow R \longrightarrow F \longrightarrow A \longrightarrow 0
\end{aligned}
$$

where $F_{j}$ is the free $A_{j}$-module with the elements of $A_{j}$ as $A_{j}$-basis and

Received December 19, 1957. 
$F_{j} \longrightarrow A_{j}$ is the identity on the basis of $F_{j}$. Similarly for $F$. It is then easy to see that $R$ may be regarded as the direct limit of the $R_{j}$ 's. If $n=\sup .1 \operatorname{dim}_{\Lambda_{j}} A_{j}$, $n>0$ then

$$
\text { sup. } 1 . \operatorname{dim}_{\Lambda_{j}} R_{j} \leqq n-1 .
$$

Since also $1 \cdot \operatorname{dim}_{\Lambda} A \leqq 1+1 \cdot \operatorname{dim}_{\Lambda} R$, it suffices to prove that $1 \cdot \operatorname{dim}_{\Lambda} R \leqq 1+(n-1)$. This reduces the theorem dy induction to the case $n=0$ i.e. to the case when each $A_{j}$ is $A_{j}$-projective.

If each $A_{j}$ is $A_{j}$-projective, then $B_{j}=\Lambda \otimes_{\Lambda_{j}} A_{j}$ is $A$-projective. The $B_{j}$ 's form a direct system of $A$-modules with $A$ as limit. This further reduces the theorem to the case when the direct system $\left\{\Lambda_{j}, \varphi_{i j}\right\}$ is constant, i.e. $\Lambda_{j}=A$, $\varphi_{i j}=$ identity.

The above two reductions are valid for any indexing set $J$. Now we assume that $J$ is countable. There exists then a sequence $a_{1}<a_{2}<\ldots<a_{k}<\ldots$ in $J$ which is cofinal with $J$. Thus we may assume that $J=(1,2, \ldots, k, \ldots)$.

Thus the direct system $\left\{A_{j}, \psi_{i j}\right\}$ is a direct sequence

$$
A_{1} \stackrel{\psi^{2}, 1}{\longrightarrow} A_{2} \stackrel{\psi^{\prime}, 2,2}{\longrightarrow} \ldots \stackrel{\psi^{\prime k, k-1}}{\longrightarrow} A_{k} \stackrel{\eta / k+1, k}{\longrightarrow} \ldots
$$

where each $A_{k}$ is a projective $\Lambda$-module. By definition, the limit $A$ is the quotient of the direct sum $B=\sum_{k=1}^{\infty} A_{k}$ by the submodule generated by all the elements $a-\psi_{k+1, k}(a), a \in A_{k}$. There results the exact sequence

$$
0 \longrightarrow B \stackrel{\gamma}{\longrightarrow} B \longrightarrow A \longrightarrow 0
$$

where $\gamma(a)=a-\psi_{k+1, k}(a)$ for $a \in A_{k}$. Since $B$ is $A$-projective, it follows that 1. $\operatorname{dim}_{\Lambda} A \leqq 1$.

\section{BiBLIOGRAPHY}

[1] V. M. Kuročkin, The decomposition of algebras into a semi-direct sum of the radical and a semi-simple subalgebra, C. R. (Doklady) Acad. Sci., URSS (N.S.) vol. 36 (1942), pp. $42-45$.

[2] A. Rosenberg and D. Zelinsky, Cohomology of infinite algebras, Trans. Amer. Math. Soc., vol. 82 (1956), pp. 85-98.

\section{Bucharest, Roumania}

